\title{
Metástasis en glándula parótida por tumoraciones cutáneas melanoma y no melanoma de cabeza y cuello. Pronósticoy tratamiento
}

\section{Parotid metastatic from cutaneous melanoma y no melanoma of head and neck. Prognostic and treatment}

\author{
Luis F. RIOJA*, María J. ESPIÑEIRA**, Macarena ÁVILA ${ }^{\star \star \star}$
}

Aldo LEAL**, Pedro E. ALONSO**

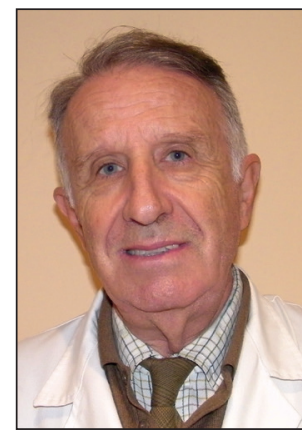

Rioja L. F.
Resumen

Introducción y objetivo. El melanoma y las tumoraciones cutáneas no melanomas (TCNM) situadas en cabeza y cuello pueden extenderse a la glándula parótida (GP), bien por continuidad o por diseminación a través del sistema linfático. Cuando esto ocurre, el tratamiento a seguir es preferentemente quirúrgico, siempre y cuando la evolución tumoral, el estado general del paciente y la no diseminación del tumor a otras zonas del organismo lo permitan.

Nuestro objetivo es analizar el tratamiento seguido, el número de recidivas y la mortalidad en nuestra serie de las citadas tumoraciones, con invasión de la GP.

Material y método. Presentamos una serie de 26 pacientes (24 varones y 2 mujeres) con afectación tumoral parotídea consecuencia de metástasis de melanoma o de TCNM. A todos se les realizó tratamiento quirúrgico, parotidectomía del lóbulo superficial en 5 casos y parotidectomía total en 21 . La disección cervical radical modificada tipo III se efectuó en 19 pacientes. Posteriormente siguieron tratamiento radio y/o quimio o inmunoterápico.

Resultados. Desarrollamos el estudio desde 2012 a 2018, con un seguimiento de los pacientes de 0 a 114 meses, encontrando un grado de recidiva del $15.38 \%$ y una mortalidad del $34.6 \%$. La complicación más frecuente como consecuencia de la cirugía ablativa realizada fue la parálisis facial en los 3 casos en que no se preservó el nervio facial y la neuropraxia del nervio facial, principalmente de sus ramas bucal y marginal, que cedió con el tiempo.

Conclusiones. El tratamiento de las tumoraciones metastásicas de la GP consecuencia de este tipo de tumoraciones cutáneas, es preferentemente quirúrgico con exéresis de la glándula y respetando el nervio facial siempre que la invasión tumoral no lo afecte. La radioterapia postoperatoria será también útil como complemento del tratamiento. Los resultados con el tratamiento combinado son actualmente poco esperanzadores, pero se espera una mejoría de las expectativas principalmente por los tratamientos inmunoterápicos en el caso de los melanomas y radioterápicos en las invasiones por carcinomas espinocelulares. Seguramente todo ello permitirá que el tratamiento quirúrgico sea menos radical y con secuelas escasas.

Palabras clave Melanoma, Carcinomas no melanoma, Tumores cabeza y cuello, Glándula parótida, Metástasis parótida

\section{Nivel de evidencia científica Recibido [esta versión] Aceptado} $4 c$ Terapéutico 15 marzo/2019

\begin{abstract}
Background and objective. When developed on head and neck, both melanoma and non-melanoma skin cancer (NMSC) can be spread to the parotid gland (PG) because of its permanence or due to the lymphatic spreading. In this case, the most appropriate option is the surgical procedure, provided that the progression of the tumor, the general condition of the patient and the non-dissemination of the tumor allow it.

Our aim is to analyze the treatment followed and the number of recurrences, as well as mortality of our series in which the tumors cited invades the GP.

Methods. The study includes a selection of 26 patients ( 22 male and $2 \mathrm{fe}$ male) with a parotid gland tumor diagnosis as a consequence of a melanoma/ NMSC metastasis. All of them had undergone surgical procedure, 5 with a superficial lobe parotidectomy, and the remaining 19 with a total parotidectomy. Modified radical neck dissection Type III was applied to 19 patients. After that, they continued receiving radiotherapy and/or chemo o inmunotherapy.

Results. This study was conducted between 2012 and 2018, with a patient's follow-up from 0 to 114 months. Patients had a recurrence rate of $15.38 \%$ a mortality of $34.6 \%$. The most frequent complications as a result of the surgical ablation were the facial paralysis in all 3 cases where the facial nerve was not preserved, and the neurapraxia in the facial nerve, specifically the marginal mandibular branches and the buccal branches, which decreased over time.

Conclusions. The preferred treatment of metastasis of PG tumors as a consequence of this kind of skin cancer is the surgical procedure with gland exeresis and keeping the facial nerve, as long as the tumor invasion does not affect it. Postoperative radiotherapy will also be an essentially useful resource as a treatment complement. For the moment, the results of the combinated therapy are not encouraging. However, thanks to the immunotherapies applied in melanoma cases and to the radiotherapy applied in squamous cell carcinoma invasions, best prospects are expected. Probably, this will result in less radical surgical procedures with few sequelae.
\end{abstract}

Key words Melanoma. No melanoma cutaneous carcinoma, Head and neck tumors, Parotid gland, Parotid metastasis

Level of evidence
Received [this version]
Accepted

$4 c$ Therapeutic

$15 \mathrm{March} / 2019$

26 November $/ 2019$

Conflicto de intereses: Los autores declaran no tener ningún interés financiero relacionado con el contenido de este artículo. Financiación: No hubo fuentes externas de financiación para este trabajo. 


\section{Introducción}

La glándula parótida (GP) es origen de tumoraciones malignas pero también una zona anatómica en la que por su riqueza ganglionar linfática, metastatizan los tumores faciales y del cuero cabelludo, principalmente los carcinomas espinocelulares (CEC) y los melanomas malignos (MM).

El cáncer cutáneo es el más común en la población de raza blanca debido a la influencia de la radiación solar. En España, la incidencia anual de este tipo de tumores es del 8.76 (IC 95\%: 7.50-10.02)/100.000 habitantes/año en el caso del MM y del 38.16 (IC 95\%: 31.72-39.97)/100.000 habitantes/año en el del CEC y del 113.05 (IC 95\%: 89.03-137.08)/100.000 habitantes/año en el $\mathrm{CBC}$, ${ }^{(1)}$ lo que supone que en España anualmente aparecerían 4.030 nuevos casos de MM, 14.591 de CEC y 52.003 de carcinoma basocelular (CBC).

La GP contiene entre 20 y 30 ganglios linfáticos intra o paraparotideos, a los que drena la linfa de la frente, de la raíz nasal, de la región periorbitaria, de la mejilla, del labio superior y de la región auricular, incluido el conducto auditivo externo. ${ }^{(2)}$

La incidencia en cabeza y cuello del CEC es del $53.49 \%$, la del CBC del $92.6 \%$ y la del MM del $17.9 \%$ del total de cuerpo. ${ }^{(3,4)}$ Los tumores de cabeza y cuello que metastatizan por vía linfática son aproximadamente el 5\% de los mismos en el área de la GP. ${ }^{(5-7)}$ En el caso del CBC la invasión se realiza por continuidad del propio tumor. La diseminación por vía hematógena es muy infrecuente en este tipo de tumores.

Nuestro objetivo es estudiar una serie de pacientes que presentan metástasis en la GP y en ocasiones también en cadenas ganglionares cervicales, siguiendo un procedimiento uniforme en el tratamiento quirúrgico $\mathrm{y}$ siendo remitidos posteriormente a radio, quimio e inmunoterapia, así como comprobar el resultado en cuanto a recidivas locales y generales, las muertes habidas y el tiempo de supervivencia de los pacientes. El pronóstico tanto de los CEC, CBC, como de los MM se ensombrece si presentan metástasis en la GP; por tanto será muy importante la detección precoz de las metástasis incipientes para aumentar la supervivencia.

La relación de la GP con en nervio facial (NF) es desde el periodo embriológico muy estrecha, y de hecho divide la glándula en dos lóbulos, superficial y profundo. En general, las metástasis linfáticas en la GP se encuentran más frecuentemente en el lóbulo superficial, aunque el tratamiento quirúrgico será de parotidectomía total y si existen metástasis en cuello se efectuará una disección del mismo. ${ }^{(8,9)}$

\section{Material y método}

Realizamos un estudio retrospectivo entre los años 2012 y 2018 de 26 pacientes que sufrieron metástasis de un tumor primario MM o un tumor cutáneo no melanoma (TCNM) de la región facial y cuero cabelludo en la GP y en ocasiones en los ganglios cervicales (Tabla I). En todos los casos constatamos que anteriormente habían padecido un tumor primario; el $82 \%$ eran pacientes tratados por nosotros y el resto, $18 \%$, acudieron procedentes de otros Servicios de nuestro hospital y de otros hospitales pertenecientes a nuestra área sanitaria.

\section{Resultados}

Se trataron 24 varones $(92.3 \%)$ con una edad media de 70.5 años (rango de 22-93 años) y 2 mujeres (7.7\%) con una edad media de 82 años (rango de 78-86 años). La edad media del conjunto de la serie fue de 71.42 años (rango de 22-93 años). Por tanto se trata de una población envejecida, en la que 19 pacientes (73\%) tienen una edad superior a los 70 años.

La naturaleza del tumor primario fue de 3 tipos: CEC, CBC y MM. El porcentaje de afectaciones parotídeas fue en el caso del CEC del 69.2\% (18 casos), seguido del MM con un $23 \%$ (6 casos) y solamente un $7.6 \%$ (2 casos) para el CBC. En los casos de afectación por CBC lo fue por continuidad y no por diseminación linfática, por tanto podría no considerarse como una metástasis parotídea (Tabla II).

La localización más frecuente del tumor primario fue la preauricular con 7 casos (26.9\%), seguida por la témporo-frontal con 7 casos (26.9\%); las otras localizaciones se dieron en menos casos (Tabla III).

Los pacientes fueron estudiados previamente a la instauración del tratamiento mediante pruebas de imagen (ecografía), citología mediante aspiración con aguja fina (PAF), tomografía computarizada (TAC), resonancia magnética (RM) y tomografía por emisión de positrones (PET), a fin de confirmar la existencia de extensión a la GP y a los ganglios cervicales. El estudio de Anatomía Patológica postoperatoria confirmó la naturaleza del tumor.

El tratamiento quirúrgico seguido fue parotidectomía superficial en 5 pacientes (19.2\%), parotidectomía total en $21(80.8 \%)$ y vaciamiento cervical radical (VCR) en 19 pacientes (73\%). En todos los casos se preservó el NF, salvo en 1 paciente con recidiva del CBC que había invadido la GP. (Fig. 1-7). La lesión del NF se produjo en 3 pacientes: en 1 que presentó invasión de la GP por $\mathrm{CBC}$ porque la tumoración ya contactaba con el nervio 


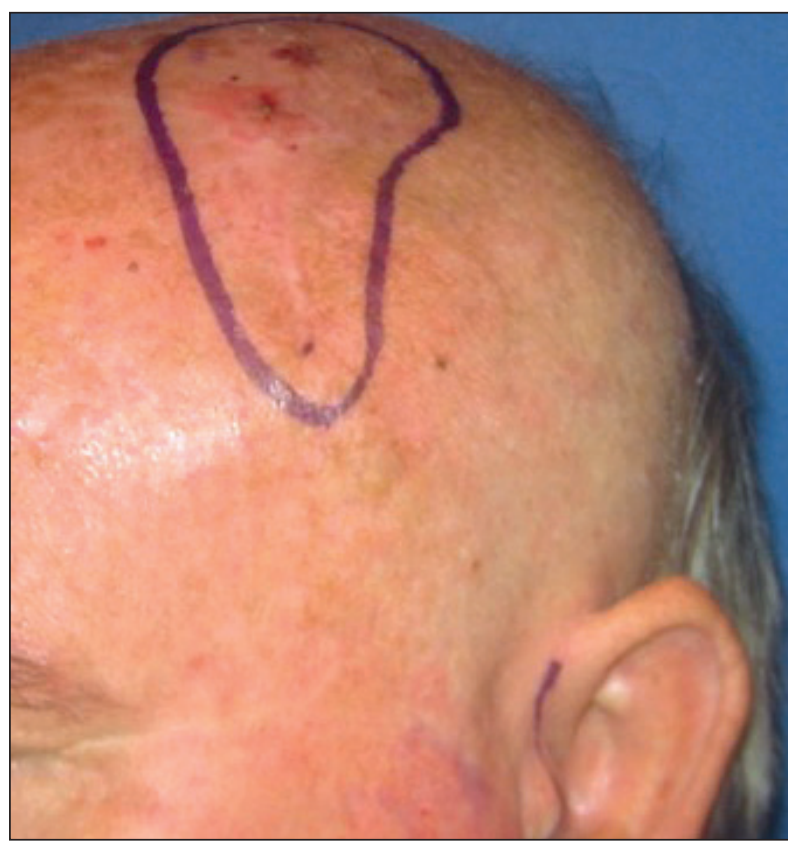

Figura. 1. Carcinoma espinocelular en cuero cabelludo que se extiende a la glándula parótida.

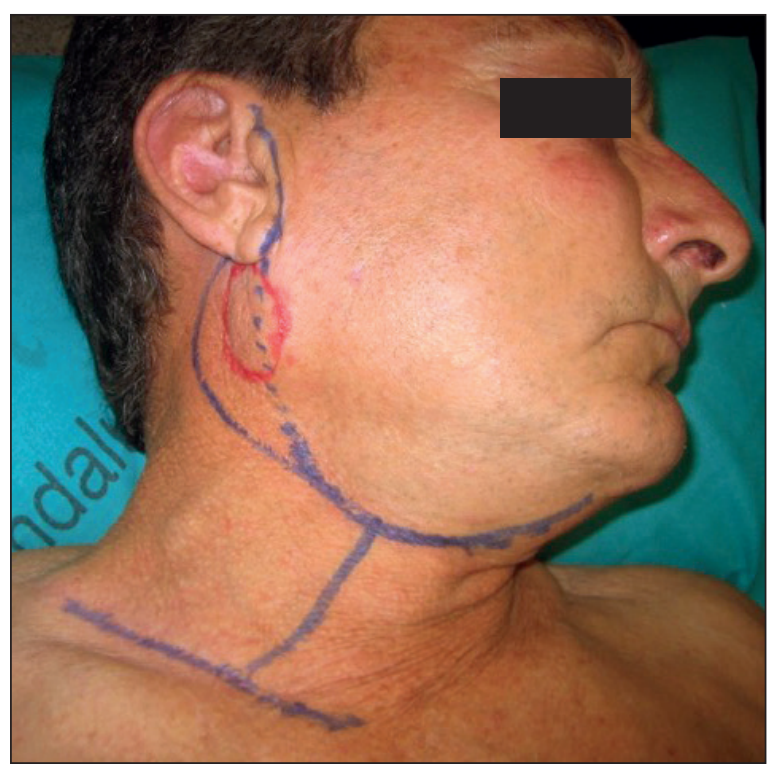

Figura. 3. Recidiva de carcinoma espinocelular del pabellón auricular en la cola de la glándula parótida. Marcas para efectuar la parotidectomía y el vaciamiento cervical radical.

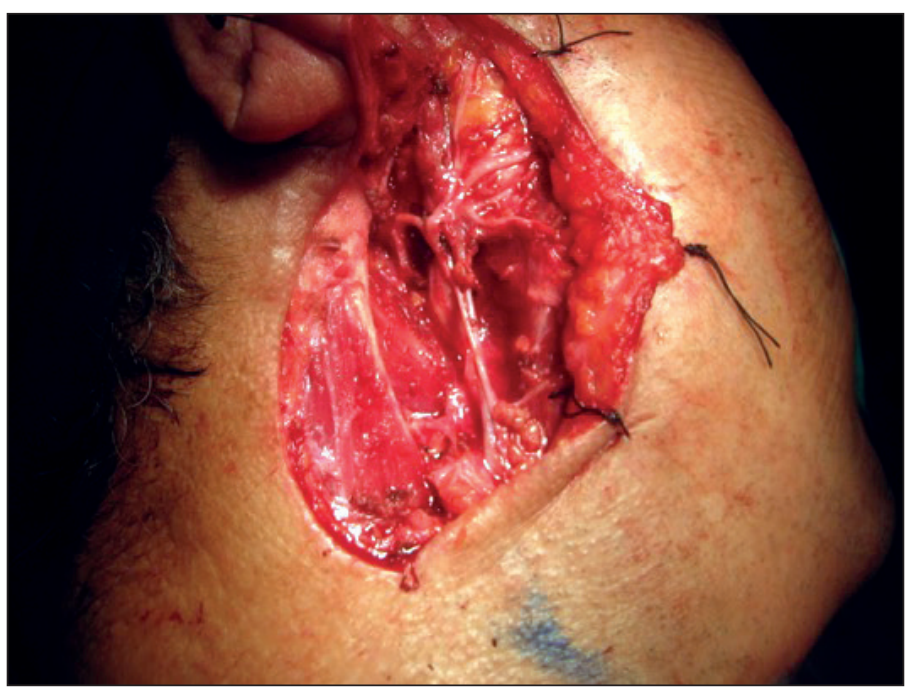

Figura. 5. Exposición del nervio facial después de la parotidectomía superficial y profunda. tida.

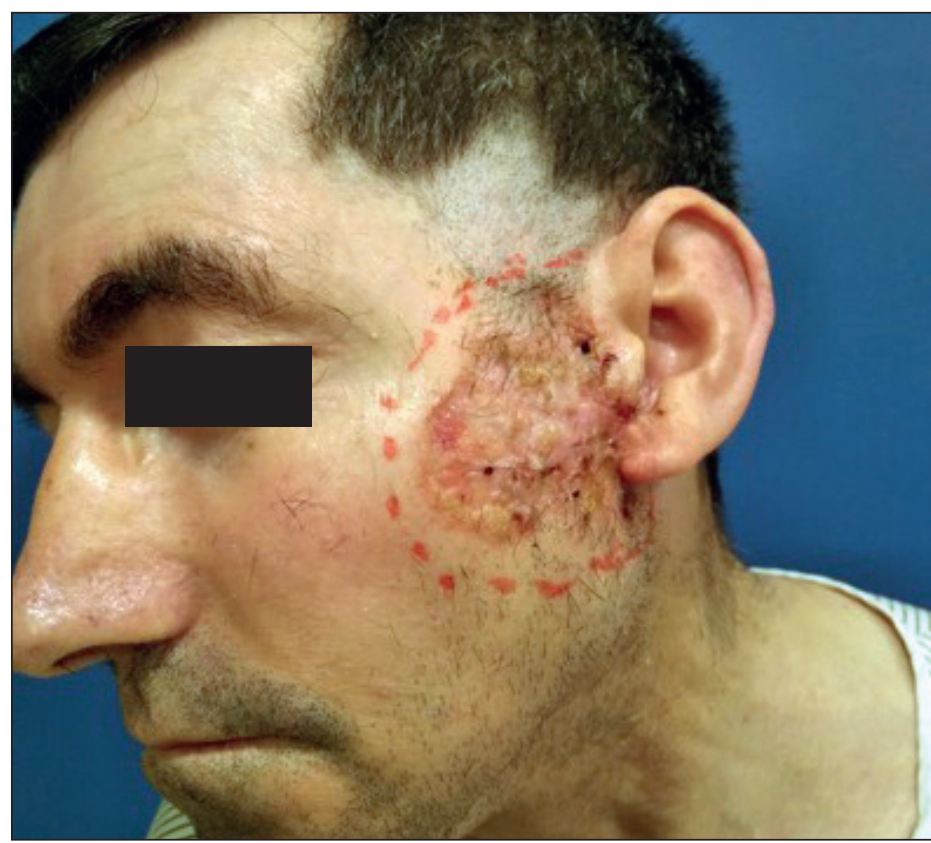

Figura. 2. Carcinoma basocelular que invade en profundidad la glándula paró-

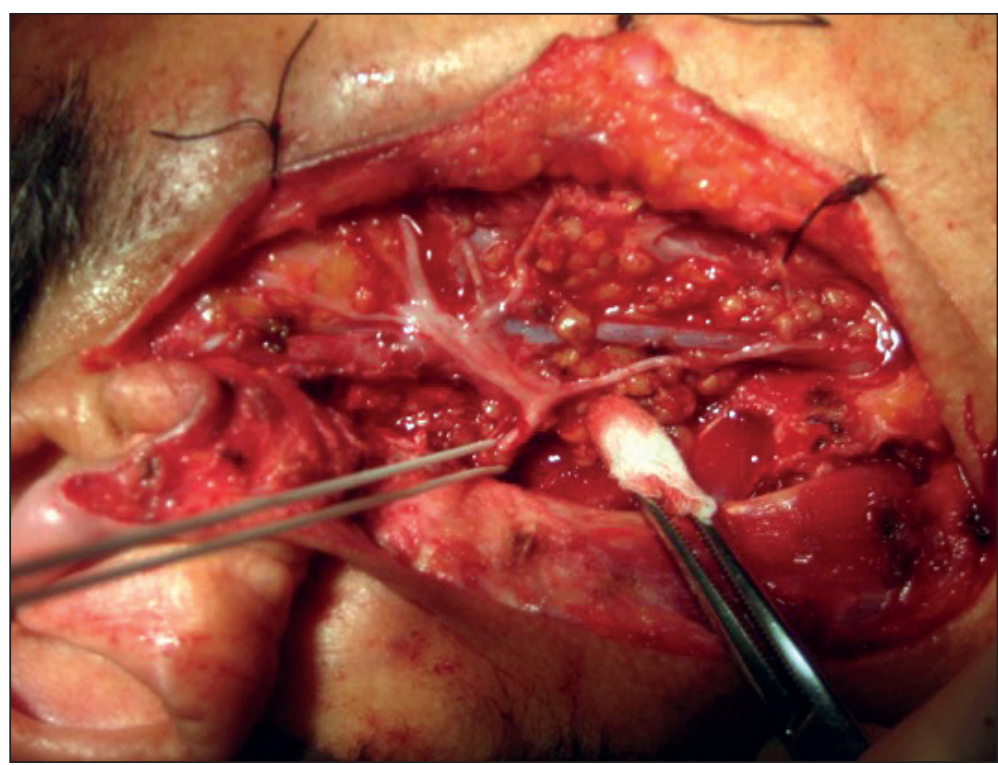

Figura. 4. Exposición del tronco del nervio facial y de sus ramas después de la paro-

tidectomía superficial.

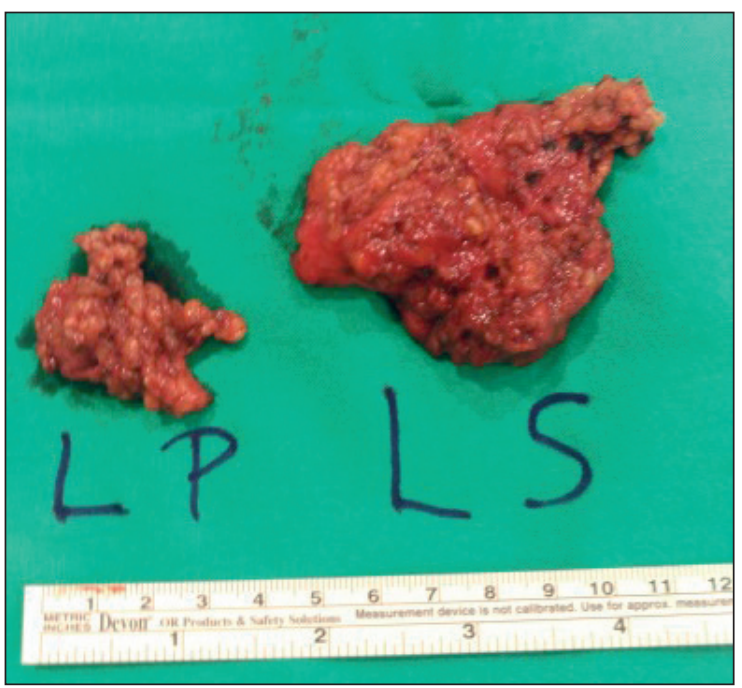

Figura. 6. Lóbulos parotídeos profundo y superficial. 


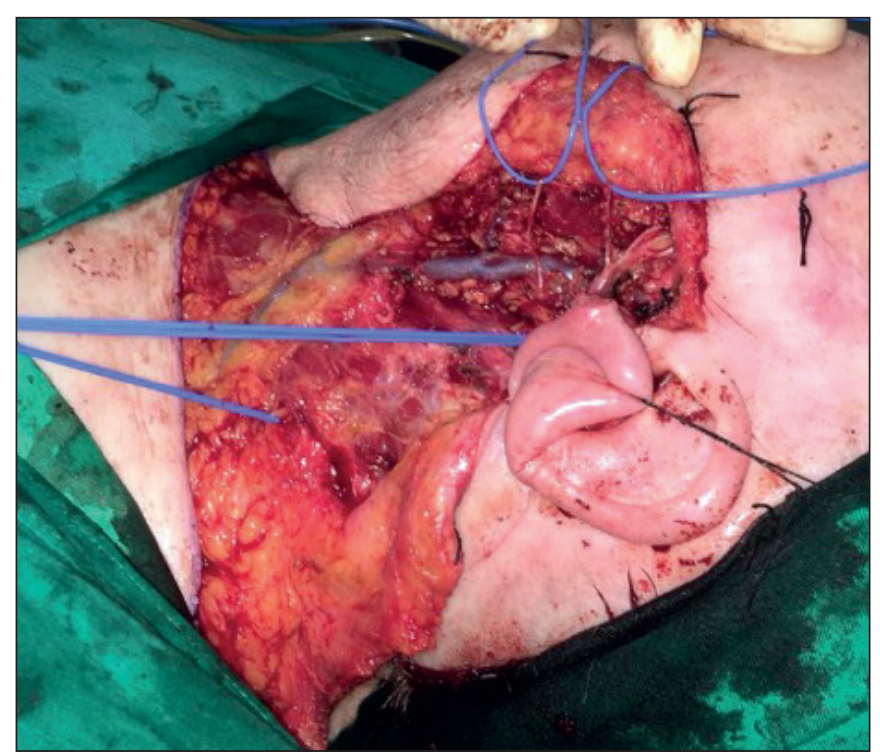

FIG. 7. Parotidectomía realizada y comienzo del abordaje del vaciamiento cervical radical. y se efectuó su inclusión dentro de la pieza tumoral; y los otros 2 casos fueron por lesión de la rama témporo-frontal en uno y de la marginal mandibular en el otro. En ambos se llegó a la recuperación parcial de la función de la comisura bucal y del cierre palpebral. El cierre de la herida post-tumorectomía se efectuó directamente sin necesidad de emplear colgajos, salvo en 3 ocasiones en las que se utilizó, en 2 casos el colgajo cérvico-torácico y en el caso del CBC de la Fig.2 el colgajo fasciocutáneo libre ánterolateral de muslo.

El VCR modificado tipo III, preservando el nervio espinal accesorio, el músculo esternocleidomastoideo y la vena yugular interna, se llevó a cabo por la aparición de adenopatías sospechosas de invasión tumoral, refrendadas por los procedimientos de imagen. En uno de los casos, el número 23, la recidiva en la GP estaba muy instaurada pero en la TAC no se detectaban me-

Tabla I. Resumen de datos de los pacientes del grupo de estudio

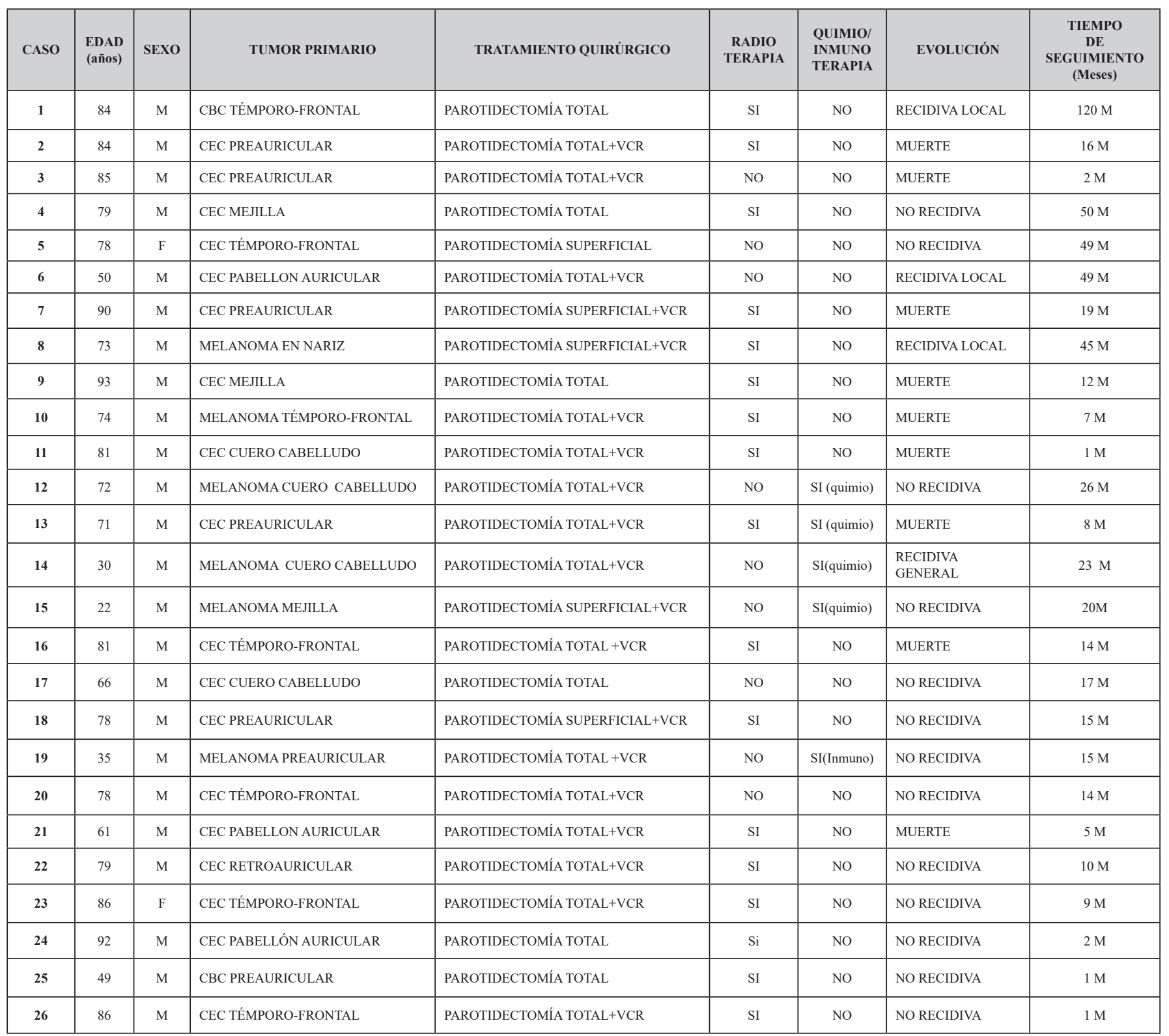


tástasis cervicales. Dada la edad avanzada del paciente (92 años), se decidió no prolongar el tiempo quirúrgico con un VCR; no obstante, el paciente sufrió en el postoperatorio un estado confusional del que lentamente fue recuperándose.

Todos los pacientes fueron remitidos a Oncología Médica y/o Oncología Radioterápica donde fueron tratados en base al tipo de tumor, grado de malignidad y extensión de las metástasis. Recibieron tratamiento radioterápico 12 pacientes $(46.15 \%)$, quimioterápico 4 pacientes (15.38\%) e inmunoterápico 1 paciente (3.84\%).

Los que tuvieron recidiva fueron $4(15.38 \%)$, de los que en 3 fue local, y el total de fallecidos fue de 9 (34.6\%).

Tabla II. Análisis de pacientes tratados por metástasis de carcinoma espinocelular, carcinoma basocelular y melanoma en la glándula parótida

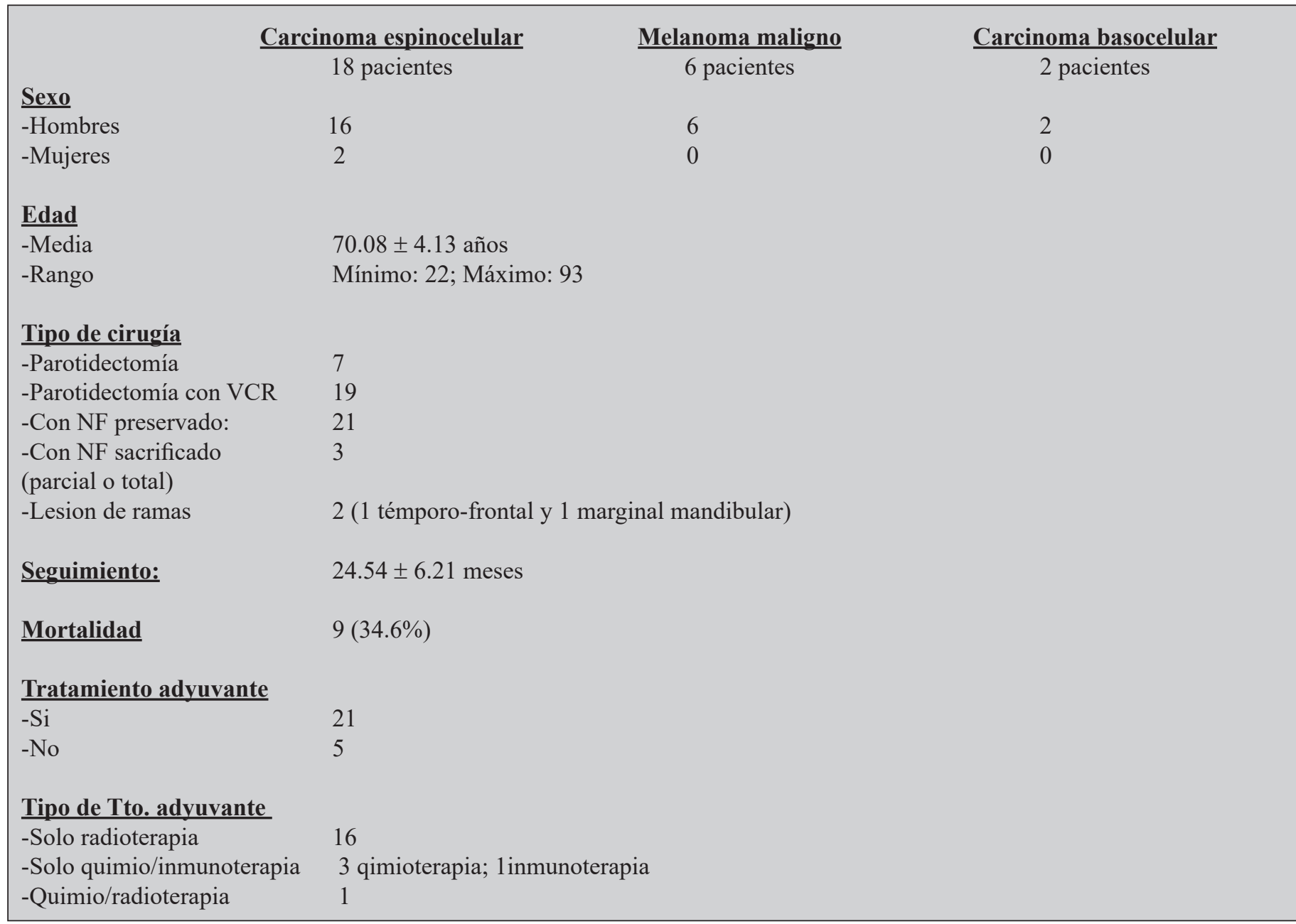

Tabla III. Localización anatómica del tumor primario

\begin{tabular}{|c|c|c|c|}
\hline & CEC & $\mathbf{M M}$ & CBC \\
\hline Cuero Cabelludo & 2 & 2 & 0 \\
\hline Región témporo-frontal & 5 & 1 & 1 \\
\hline Pabellón auricular & 3 & 0 & 0 \\
\hline Región preauricular & 6 & 1 & 1 \\
\hline Mejilla & 2 & 1 & 0 \\
\hline Región nasal & 0 & 1 & 0 \\
\hline
\end{tabular}

$C E C=$ Carcinoma espinocelular MM=Melanoma maligno; $C B C=$ Carcinoma basocelular

\section{Discusión}

La GP alberga tumores primarios fundamentalmente benignos, pero también es un área anatómica a la que pueden extenderse los tumores faciales y del cuero cabelludo y mucho más infrecuentemente de otras zonas del cuerpo. Si bien un estudio de Gnepp con 6.000 pacientes que presentan tumoraciones parotídeas benignas, malignas y metastásicas, ofrece una incidencia pequeña, menor del $10 \%$ del total, ${ }^{(10)}$ otros que cuantifican únicamente las tumoraciones malignas primarias y metastásicas de la GP ofrecen un porcentaje mayor. Así Bron, en una serie de 232 casos, señala que las tumoraciones primarias eran $54(23.3 \%)$ y las metastásicas $178(76.7 \%)$, de las que el 95\% correspondían a CEC y MM, si bien hay que destacar que dicho estudio se realiza en Australia y que la incidencia allí de las tumoraciones cutáneas es muy elevada. ${ }^{(11)}$ Los tumores que metastatizan más frecuentemente en la GP son los CEC 
y los MM, representando cifras cercanas al $80 \%$ de las metástasis en esta glándula. ${ }^{(12)}$

En nuestro estudio no fue imposible calcular el número de pacientes a los que se ha tratado de CEC, CBC y MM puesto que nos limitamos a recibir ese tipo de pacientes que acuden ya con la extensión del tumor en la GP, siendo un número limitado de pacientes los que desde el inicio de la tumoración son tratados íntegramente por nuestro Servicio; por tanto no podemos calcular qué porcentaje de los tumores melanoma y no melanoma afectan una vez tratados inicialmente a la GP.

Las metástasis en la GP de los tumores faciales y del cuero cabelludo son muy agresivas, y si bien la realización de una parotidectomia superficial combinada con VCR es frecuente en muchos centros hospitalarios dado que el número de ganglios linfáticos del lóbulo superficial es superior a los del lóbulo profundo aproximadamente en una proporción de 4 a $1 .^{(13-14)}$ Creemos que al no existir una verdadera barrera entre ambos lóbulos, solamente separados por las ramas del NF, la vulnerabilidad de invasión del lóbulo profundo es elevada. Hay hallazgos que han demostrado que con metástasis linfáticas en el lóbulo superficial se presentarían metástasis ocultas en el lóbulo profundo. ${ }^{(15)}$ No hemos encontrado en ningún caso metástasis aisladas en el lóbulo profundo sin que estuviera afecto el lóbulo superficial. Por tanto nos parece que el tratamiento ciertamente es más completo cuando se realiza la resección de los dos lóbulos. Si en nuestros primeros casos actuábamos muy preferentemente sobre el lóbulo superficial cuando no había constancia de metástasis en el lóbulo profundo, nuestra actitud ha ido evolucionando hacia la extirpación completa de la GP. ¿Está justificada esta cirugía radical con la GP? Pensamos que si se quiere controlar la recidiva local en dicha glándula es importante la eliminación completa de los ganglios que se encuentran en ella. En nuestra serie hubo 4 recidivas, 3 locales y 1 general que no ocasionaron la muerte de los pacientes, aunque registramos 7 casos de fallecimiento por diseminación del tumor y 2 por otras causas.

La preservación del NF se hace por tanto inevitable; en ningún caso hemos encontrado un atrapamiento de las ramas del nervio facial cuando las metástasis han sido ganglionares, únicamente en el caso del CBC, en que se produjo una invasión glandular por el mismo tumor, existía una afectación de varias ramas del NF, no así del tronco.

El número total de muertes contabilizado en nuestra serie fue de 9 (34.61\%); aunque la mortalidad en el primer año después de la cirugía parotídea fue de 6 pacientes, 5 de ellos con CEC y 1 con MM, esta mortalidad es menor que la de otras series en las que posiblemen- te, por aportar un mayor número de MM respecto a los carcinomas epidermoides, se altera negativamente dicha mortalidad. ${ }^{(16)}$

La presencia en nuestra serie de 2 casos de CBC que invaden la GP por continuidad y no por extensión del sistema linfático, hace que puedan no considerarse como metastásicos. ${ }^{(17)}$ En estos casos, el procedimiento quirúrgico fue la parotidectomía total, y en 1 de los caso se sacrificó la rama témporo-frontal del NF que estaba invadida por la tumoración.

En los supuestos de invasión de la GP por tumoraciones cutáneas melanoma o no melanoma, consideramos que la cirugía radical sobre la GP es muy conveniente para el control de las recidivas locales en esa área y por tanto, para controlar más eficazmente al tumor y la progresión de la enfermedad.

Los recientes tratamientos inmunoterápicos han de constituir una esperanza para el control de este tipo de extensiones tumorales.

\section{Conclusiones}

Las tumoraciones cutáneas melanoma y no melanomas son las más frecuentes del organismo. Las metástasis en la glándula parótida de estas tumoraciones cuando se localizan en el área de cabeza y cuello, son siempre un factor pronóstico negativo.

Es importante el diagnóstico precoz en este tipo de tumoraciones para evitar su extensión. De igual modo, la precocidad del diagnóstico y tratamiento una vez que se localiza la metástasis en la glándula parótida, va a mejorar el pronóstico de supervivencia de los pacientes.

Cuando la invasión llega a los ganglios cervicales la supervivencia empeora.

En la actualidad la inmunoterapia, utilizada principalmente en los melanomas, abre un camino de esperanza para una mejor supervivencia.

\section{Dirección del autor}

Dr. Luis Fernando Rioja Torrejón

Servicio de Cirugía Plástica

Hospital Universitario Reina Sofía

Avda. Menéndez Pidal S/N

CP 14004, Córdoba, España

Correo electrónico: ferlu8791@hotmail.com

\section{Bibliografía}

1. Tejera-Vaquerizo A, Descalzo-Gallego MA, Otero-RivasMM, et al. Incidencia y mortalidad del cáncer cutáneo en España: Revisión sistemática y metaanálisis. Actas Dermo-Sifilograficas. 2016; 107(4):318-328. 
2. Batsakis JG. Parotid gland and its Lymph nodes as metastatic sites. Ann OtolRhinolLaryngol. 1983; 92:209-210.

3. Schwart R, Schwart R. Cáncer de piel no melanoma en cabeza y cuello. Rev Médica Clínica Las Condes. 2018; 29:455-467.

4. Hinerman RW, Indelicato DJ, Amdur RJ, Et Al. Cutaneous squamous cell carcinoma metastatic to parotid-area lymph nodes. Laryngoscope 2008; 118:1989-1896.

5. Vauterin TJ, Veness RS, Morgan GP, Poulsen MG, O'Brien CJ. Patterns of lymph node spread of cutaneous squamous cell carcinoma of the head and neck. Head Neck. 2006; 28(9):785791.

6. Moore BA, Weber RS, Prieto V. Et al. Lymph node metastases from cutaneous squamous cell carcinoma of the head and neck. Laryngoscope. 2005; 115(9):1561-1567.

7. Pathak I, O'Brien CJ, Petersen K, et al. Do nodal metastases from cutaneous melanoma of the head and neck follow a clinically predictable pattern? Head Neck. 2001; 23(9):785-790.

8. AndruchowJL, Veness MJ, Morgan GJ, et al. Implications for clinical staging of metastatic cutaneous squamous carcinoma of the head and neck base don a multicenter study of treatment outcomes. Cancer. 2006; 106(5):1078-1083.

9. O'Hara J, Ferlito A, Takes RP, et al. Cutaneous squamous cell carcinoma of the head and neck metastasizing to the parotid gland: a review of current recommendations. Head Neck. 2011; 33(12):1789-1795
10. Gnepp DR. Metastatic disease to the major salivary glands. En: Ellis GL, Auclair Pl, GneppD.: Surgical Pathology of the salivary glands. WB Saunders Company, Philadelphia, PA (USA) 1991: 560-569.

11. Bron LP, Traynor SJ, McNeil EB, O'Brien CJ. Primary and metastatic cancer of the parotid: Comparison of clinical behavior in 232 cases. Laryngoscope. 2003; 113:1070-1075.

12. Coulthard SW. Metastatic disease of the parotid gland. Otolaryngol Clin Noth Am 1977; 10(2):437-442.

13. Pisani P, Ramponi A, Pla F. The Deep parotid Lymph nodes an anatomical and oncological study. J LaryngolOtol. 1996; 110(2):148-150.

14. Garatea Orelgo J, Gay Escoda C, Bermejo B, BuenecheaImaz R. Morphological study of the parotid lymph nodes. J CraniomaxilofacSurg. 1993; 21(5):207-209.

15. Thom JJ, Moore EJ, Price DL, Kasperbauer JL, Starkman SJ, Olsen KD. The role of total Parotidectomy for metastatic Cutaneous Squamous cell carcinoma and malignant Melanoma. Jama Otolaryngol. Head Neck Surg. 2014; 140(6):548-554.

16. Jecker P, Hartwein J. Metastasis to the Parotid Gland: Is a Radical Surgical Approach Justified?.Am J Otolaryngol. 1996; 17(2):102-105.

17. Pérez de la Fuente T, González González I. Carcinoma basocelular metastásico en la región parotídea: un nuevo caso. Cir plást. iberolatinoam.2006; 32(1):63-67. 
